\title{
Holtkamp, Lars: Der Parteienstreit. Probleme und Reformen der Parteiendemokratie, 130 S., Nomos, Baden-Baden 2018.
}

\author{
Karlheinz Niclauß \\ Online publiziert: 5. Juni 2020 \\ (C) Der/die Autor(en) 2020
}

Der Titel „Parteienstreit“ bezieht sich, wie Lars Holtkamp einleitend erläutert, nicht auf den Streit zwischen den Parteien in der Bundesrepublik, sondern auf den Streit zwischen den Parteienforschern. In diesem Streit stehen sich nach Auffassung Holtkamps Parteikritiker wie Hans Hermann von Arnim und die ,traditionelle politikwissenschaftliche Parteienforschung“ gegenüber. Während von Arnim die Fehlentwicklungen im deutschen Parteiensystem analysiert, argumentiere die traditionelle Parteienforschung affirmativ und leugne außerdem die unterschiedliche Entwicklung zwischen Ost und West in Deutschland. Der Autor versucht, diesen Streit durch eine Untersuchung des deutschen Parteiensystems zu schlichten. Als Leitlinie dient ihm hierbei ein „,parteipolitischer Repräsentationszyklus“, der von einer starken Verankerung der Parteien in der Gesellschaft ausgeht. Die Parteien entwickeln demnach unter Mitwirkung ihrer Mitglieder „klare inhaltliche Regierungsalternativen“. Auf dieser Grundlage führen die allgemeinen Wahlen zu Regierungsentscheidungen ,gemäß Parteiprogramm“, die anschließend mithilfe von Patronage umgesetzt werden.

In seiner Darstellung des deutschen Parteiensystems, die den Hauptteil seines Buches bildet, konstatiert Holtkamp zunächst eine zunehmende „Parteiverdrossenheit“, die sich in einer sinkenden Wahlbeteiligung und im Rückgang der Mitgliederzahlen äußert. Im Abschnitt über die Kartellbildung im Parteiensystem hätte man eine ausführliche Einschätzung der von 1966 bis 1969 und seit 2005 (mit Unterbrechung) regierenden Großen Koalition erwartet. Stattdesssen unternimmt Holtkamp hier einen Exkurs in die Kölner Kommunalpolitik. Die dortige Kartellbildung in Form des „Kölschen Klüngels“ ist aber durch die Veröffentlichungen von Ute und Erwin K. Scheuch seit 1994 bekannt und für die Bundesebene wenig erhellend. Überzeugender ist dagegen Holtkamps negativer Befund zur innerparteilichen Demokratie: Mitgliederorganisation, Parteiapparat und Führungszirkel hätten sich entkoppelt und führten ein Eigenleben. Die Vorauswahl der Kandidaten für die allgemeinen Wahlen wird demnach in den Parteien von einer ,dezentralisierten Oligarchie“ getroffen. Das personalisierte Verhältniswahlrecht biete anschließend dem Wähler oberhalb der kommunalen Ebene kaum Möglichkeiten zur Kandidatenauswahl. In einem System

K. Niclauß $(\bowtie)$

Universität Bonn, Bonn, Deutschland

E-Mail: ups510@uni-bonn.de 
von sieben Parteien (Holtkamp spricht von sechs) könne der Wähler die Zusammensetzung der Regierung nicht mehr bestimmen.

Die Umsetzung von Parteiprogrammen in die politische Praxis wird im deutschen politischen System durch eine Vielzahl von Vetospielern behindert. Holtkamp nennt als Beispiele den Bundesrat, die Europäische Union, das Verfassungsgericht und die Schuldenbremse. Hinzu komme die „Präsidentialisierung der deutschen Parteiendemokratie“: Kanzlerin oder Kanzler dominierten die politischen Entscheidungen ohne Rücksicht auf die Meinung der Parteien. Hartz IV und die Flüchtlingskrise offenbarten diese „Repräsentationslücken“, bei denen die Parteien nicht in der Lage waren, ihre Kanzler wieder ,einzufangen“. Holtkamp untersucht auch das AfD-Ergebnis bei der Bundestagwahl 2017. Es bestätigt seiner Ansicht nach, dass in den neuen Bundesländern eine politische Sonderentwicklung vorliegt, was die Parteienforschung angeblich bestreitet. Der Erfolg der populistischen Rechtspartei widerspricht allerdings seiner These von einer generellen Parteiverdrossenheit.

Die Bestandsaufnahme zeigt nach Holtkamp, dass sein zu Beginn vorgestellter „parteipolitischer Repräsentationszyklus“ gestört ist. Sein Schlusskapitel gilt daher Reformvorschlägen, die er allerdings mit der gebotenen Vorsicht beurteilt: In Volksbegehren und Volksentscheiden sieht er zu Recht die Gefahr neuer Vetopositionen. Sie sollten deshalb durch hohe Hürden auf Ausnahmen begrenzt werden. Einen obligatorischen Volksentscheid befürwortet er jedoch für den Fall, dass sich Bundestag und Bundesrat bei einer Gesetzesvorlage nicht einigen können. Die Direktwahl der Ministerpräsidenten in den Ländern lehnt er ab, weil dies zum Konflikt zwischen dem Regierungschef und einer politisch anders ausgerichteten Landtagsmehrheit führen könnte. Kumulieren und Panaschieren hält er auch auf Bundes- und Landesebene für sinnvoll, weil hierdurch tatsächlich eine Personenwahl ermöglicht werde. Instrumente der „kooperativen Demokratie“, wie die sogenannte Planungszelle oder Runde Tische, spielen in der Kommunalpolitik eine Rolle, haben aber auch hier nur beratende Bedeutung.

Insgesamt hat Holtkamp einen anregenden Beitrag zum deutschen Parteiensystem vorgelegt. Seine Gedankenführung leidet aber unter der Fixierung auf den angeblichen Konflikt zwischen den Parteiforschern, der in der Literatur in dieser Form kaum zu erkennen ist. Auch der Vorwurf, die Parteienforschung habe die neuen Bundesländer vernachlässigt, lässt sich kaum aufrechterhalten: Die Entwicklung von der SED zur PDS und zur Partei „Die Linke“, ihre Verfassungsmäßigkeit und die Frage ihrer Regierungsbeteiligung sind zentrale Themen der Parteienforschung. Die AfD dagegen war erst bei der Europawahl und den Landtagswahlen von 2014 erfolgreich und ist für die Parteienforschung ein vergleichsweise neues Thema. Der für die Demokratie weitaus wichtigere Streit zwischen den Parteien selbst, der mit den Großen Koalitionen weitgehend außer Kraft gesetzt ist, gerät in der Darstellung Holtkamps aus dem Blickfeld. Im Zusammenhang mit der Bundestagswahl 2017 weist er jedoch auf die „,kulturelle Konfliktlinie“ zwischen der AfD und den Grünen hin, die in der Parteienlandschaft ,entgegengesetzte Pole“ einnehmen. Dies gilt für die Flüchtlingspolitik, aber auch - wie man inzwischen hinzufügen kann - für die Klimapolitik. In einem demokratischen Parteiensystem ist dies allerdings ein Parteienstreit mit einer „schiefen Schlachtordnung“. 
Funding Open Access funding provided by Projekt DEAL.

Open Access Dieser Artikel wird unter der Creative Commons Namensnennung 4.0 International Lizenz veröffentlicht, welche die Nutzung, Vervielfältigung, Bearbeitung, Verbreitung und Wiedergabe in jeglichem Medium und Format erlaubt, sofern Sie den/die ursprünglichen Autor(en) und die Quelle ordnungsgemäß nennen, einen Link zur Creative Commons Lizenz beifügen und angeben, ob Änderungen vorgenommen wurden.

Die in diesem Artikel enthaltenen Bilder und sonstiges Drittmaterial unterliegen ebenfalls der genannten Creative Commons Lizenz, sofern sich aus der Abbildungslegende nichts anderes ergibt. Sofern das betreffende Material nicht unter der genannten Creative Commons Lizenz steht und die betreffende Handlung nicht nach gesetzlichen Vorschriften erlaubt ist, ist für die oben aufgeführten Weiterverwendungen des Materials die Einwilligung des jeweiligen Rechteinhabers einzuholen.

Weitere Details zur Lizenz entnehmen Sie bitte der Lizenzinformation auf http://creativecommons.org/ licenses/by/4.0/deed.de.

\title{
Roth, Clémentine: Why Narratives of History Matter. Serbian and Croatian Political Discourses on European Integration, 372 S., Nomos, Baden-Baden 2018.
}

\author{
Klaus Buchenau \\ Online publiziert: 8. April 2020 \\ (C) Der/die Autor(en) 2020
}

Dass Sprache eine eigene Macht hat, gilt in den Geisteswissenschaften mittlerweile als intellektuelles Allgemeingut. In den Sozialwissenschaften scheint das noch nicht überall so zu sein. Clémentine Roth nimmt sich daher in dieser 2017 an der Universität Bremen angenommenen Dissertation vor, das Studium von Narrativen und ihren Wirkungen stärker in der Politikwissenschaft zu etablieren. Sie möchte erfahren, wie Geschichtserzählungen dazu dienen, den Weg Kroatiens und Serbiens in die Europäische Union zu (de)legitimieren.

Ihre Quellen sind recht breit gestreut, Roth sucht nach historischen Bezügen in juristischen und politischen Dokumenten beider Länder, in Parteiprogrammen, Parlamentsdebatten und schulischen Geschichtsbüchern. Außerdem hat sie 19 Experteninterviews in Belgrad und Zagreb geführt. Um das Material übersichtlich zu

K. Buchenau $(\bowtie)$

Universität Regensburg, Regensburg, Deutschland

E-Mail: klaus.buchenau@geschichte.uni-regensburg.de 